\title{
Realistic models of children heads from 3D-MRI segmentation and tetrahedral mesh construction
}

\author{
Jasmine Burguet, Najib Gadi; Isabelle Bloch \\ Ecole Nationale Supérieure des Télécommunications \\ Département TSI - CNRS UMR 5141 LTCI - 46 rue Barrault, 75013 Paris, France \\ E-mail: \{jasmine.burguet,najib.gadi,isabelle.bloch\}@enst.fr
}

\begin{abstract}
In order to analyze the sensitivity of children to RF fields and mobile phones in particular, the SAR (Specific Absorption Ratio) defined as the power absorbed by a unit of mass of tissues $(\mathrm{W} / \mathrm{kg}$ ) should be computed based on a numerical model of the head. We propose to build realistic models from 3D-MRI of children heads. The method is composed of two steps. The first one consists in segmenting the main tissues in these images (skin, fat, muscles, cortical and marrow bones, cerebrospinal fluid, grey and white matter, blood, etc.). The segmentation is based on mathematical morphology methods which are well adapted to this aim and provide a robust and automatic method requiring minimum user intervention. Using simplified segmented images, the second step concerns the tetrahedral mesh generation. Our method uses almost regular meshes and topological tools to preserve the topological arrangement of the head tissues. A method to guarantee a good geometrical quality is also provided. Keywords: MRI segmentation, brain imaging, mathematical morphology, tetrahedral mesh, topological 3D labeling.
\end{abstract}

\section{Introduction}

The widespread availability of mobile phones is a fairly recent phenomenon. Their use has escalated over the past decade and, to many people, they are an essential part of business, commerce and society. There are now about 40 million mobile phones in use in France. The phones are supported by about 31000 base stations (in France), and this number will grow in the future with the development of $3 \mathrm{G}$ technology (UMTS network). Research shows that one in four Japanese children have mobile phones, in UK $52 \%$ of all seven to sixteen year-old children own a mobile phone, and

\footnotetext{
* Najib Gadi is currently with France Telecom R\&D, France.
}

$24 \%$ of young mobile users own already their third handset. Japan (and also Europe in the near future) has led the way with the development of $3 \mathrm{G}$ technology hand phones, which provide access to the web and e-mail. These phones are extremely popular among teenagers, with their enhanced graphics and in particular access to music, sport and dating web sites. Their extensive use has been accompanied by a public debate about possible adverse health effects especially for children. The main concerns relate to the emissions of radio-frequency $(\mathrm{RF})$ radiation from the phones (the handsets) and from the base stations that receive and transmit the signals and allow communication with the network.

To compute electromagnetic field propagation through the children head, using for example finite element methods, we need a head model as realistic as possible. The purpose of this paper is to generate such models. We propose to build them from real 3D magnetic resonance images (MRI). The first step developed in Section 2 concerns the segmentation of children head from MRI. The tissues of interest for the foreseen application are skin (as well as fat and muscles), cortical and marrow bones, cerebrospinal fluid (CSF), grey and white matter. Next, based on a segmented image, we introduce in Section 3 a method allowing us to build tetrahedral meshes in which each tetrahedron is labeled with one segmented structure (brain or skull for instance). Common approaches to build tetrahedral meshes use a polyhedral representation of the boundary of the objects to tessellate, then introduce points in the interior of the obtained surface, before applying a Delaunay Tesselation in order to generate the finite elements [5, 9]. One drawback is the necessity of post-treatments to remove bad quality tetrahedra, and the complexity of head structures surfaces make the application of such methods very difficult. Then we decide to use Almost Regular Tetrahedra which produce good quality meshes $[10,11]$. One originality of the labeled mesh construction is that the 
labeling procedure uses topological constraints, guaranteeing that the main tissues have a correct topology (for instance no holes on top of the skull). As the mesh quality, this is an important criteria for the validity of the numerical methods for computing electromagnetic field propagation, for which our labeled mesh will constitute the input, this step being out of the scope of this paper.

\section{Segmentation}

In order to provide realistic models, we base our approach on MR images of children heads. The evolution of head morphology (thickness of tissues, relative volume of components, etc.) necessitates to work with several images, corresponding to different ages (between 3 and 15 years old). In this section, we propose a segmentation scheme adapted to children images. It is mainly based on mathematical morphology tools.

\subsection{The Watershed Algorithm}

The watershed notion comes from the field of topography: a drop of water falling on a relief follows a descending path and eventually reaches a minimum. Watershed lines are the divide lines of the domains of attraction of drops of water. This intuitive approach is not well suited to practical implementations, and can yield biased results in some cases. An alternative approach is to imagine the surface being immersed in a lake, with holes pierced in local minima. Water will fill up basins starting at these local minima, and, at points where waters coming from different basins would meet, dams are built. As a result, the surface is partitioned into regions or basins separated by dams, called watershed lines [13].

The watershed transform has been applied in many different image processing applications and constitutes a powerful tool for segmentation since it guarantees to obtain closed contours. Several alternative definitions and calculation algorithms have been defined for the watershed transform: a complete review can be found in [1].

Its main problem is that it often provides an oversegmentation, due to the numerous regional minima usually present in an image or in a gradient image. Several methods can be used to avoid this, consisting mainly in filtering either the initial image or the image on which the watersheds are applied (typically a gradient image), or merging a posteriori the obtained regions. If some a priori knowledge on the desired segment is available, then one may employ a powerful method using markers. This is the case in our application.

\subsection{Component tree and automatic se- lection of markers}

When using markers to limit the number of the function minima before the watersheds are applied, the watershed-based segmentation techniques are usually based on the following scheme:

1. selection of markers of the objects to extract,

2. reconstruction of boundaries between the markers objects by using the watersheds.

This scheme avoids post-processing steps usually needed after watersheds. The selection of markers can be done in various ways, depending on the type of available knowledge. For our application, we exactly know the number of objects to find. Moreover, we want to define them as the most pertinent areas of the grey level function. In [4] authors proposed a new way to extract a known number of markers with a reasonable time complexity. They use a component-tree representation of a function towards this aim. The use of a tree in order to represent the "meaningful" information contained in a function is not new. In particular, Hanusse and Guillataud [6] claim that such a tree can play a central role in image segmentation, and suggest a way to compute it, based on an immersion simulation. The algorithms used to compute the component tree can be found in $[2,7]$. The last reference also contains a discussion about the time complexity of different algorithms.

\subsection{Contribution in the case of children}

In the literature all segmentation methods are designed and applied on adult images. Although our segmentation approach is largely inspired from the one developed for adult heads in [4] i.e. using mainly markers and watersheds, several specificities in case of children have to be taken into account. Our contribution in the segmentation step is mainly at this level. In particular the evolution of the tissues we want to segment has to be taken into account. For instance, for children between 0 and 8 , the bone thickness varies from 0 to $4 \mathrm{~mm}$ and remains approximately constant for ages older than 8 years. This type of prior information can be introduced as parameters of the segmentation method (mainly size of the structuring elements). Similarly the segmentation of the other structures was 
adapted in order to account of the variation in size and thickness during aging.
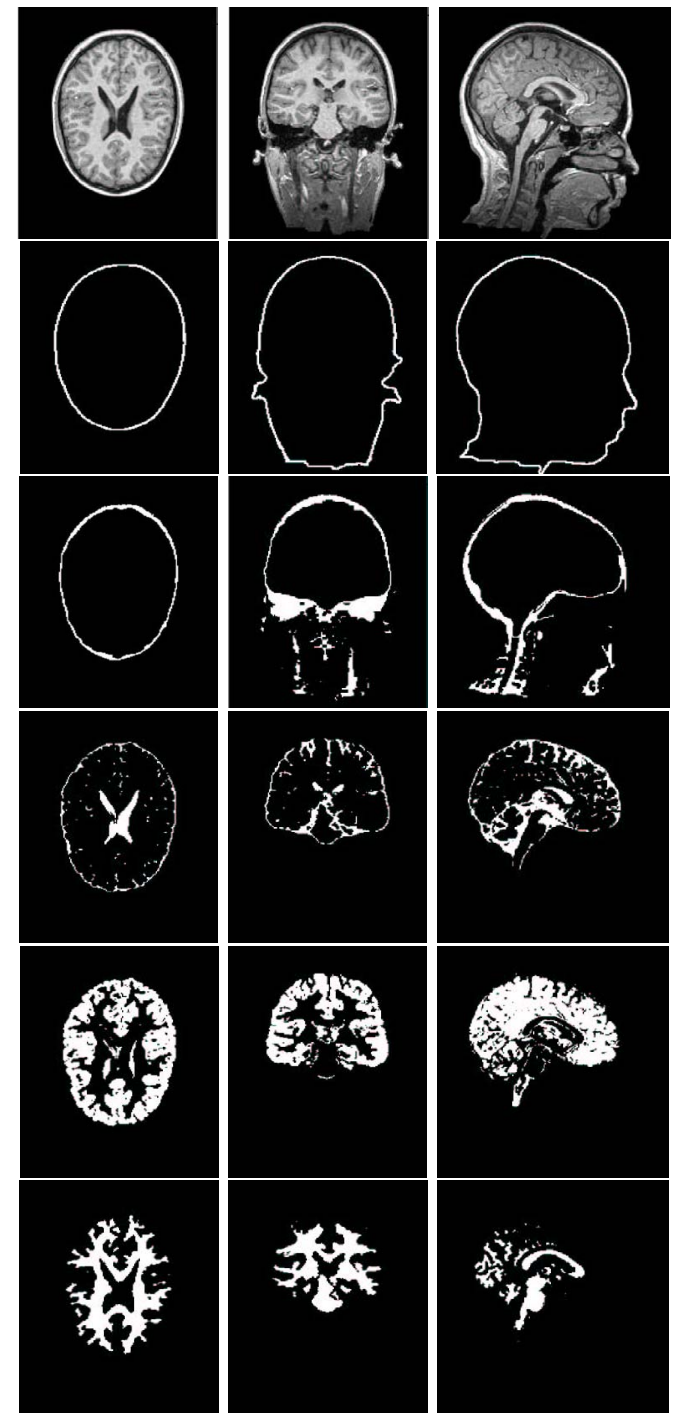

Figure 1: Three orthogonal slices (axial, coronal and sagittal) of the original image (top) and then from top to bottom: skin, skull, CSF, grey matter and white matter.

One structure requires a more detailed explanation: the CSF. While in adult heads its thickness is large enough to lead to visible thin structures in the images (with a resolution of about $1 \mathrm{~mm}$ in all directions), in child heads, the CSF is often too thin to be seen in the images. Although the CSF actually surrounds the brain such that there is no connection between brain and skull, it is difficult to incorporate this constraint without labeling erroneously skull voxels as CSF. This is a typical example where we have to abandon this strong a priori information. Our segmentation of CSF therefore does not respect the topology of this structure, but fits well the actual grey levels in the images. One advantage (and originality) of our method is that this segmentation step preserves the right morphology, while the subsequent mesh labeling step will recover the lost topology, as explained in Section 3.

\subsection{Segmentation results}

In Figure 1, three orthogonal slices of a 3D MRI are presented, as well as segmentation results of skin, skull, CSF, white and grey matters. A partition of the head is actually obtained.

The skin is well detected. It has been closed at the bottom of the head in order to guarantee a topology of empty sphere, which is a requirement of the foreseen application. The skull has also the desired topology on its top part (until the bottom of the brain). The lower part still needs some improvements in order to differentiate the air areas. The CSF is segmented according to the grey levels, and it can be seen on this results that its connectivity is sometimes lost due to it to low thickness. Its topology will be recovered in the next section. White and grey matters are well separated and satisfactory results are obtained.

\section{Tetrahedral mesh generation}

It is well accepted that interwoven spheres are a good approximation of head structures $[8,12]$. In this paper, we consider a simplified version of the segmented image computed in Section 2. Indeed, our method takes into account a topological aspect during the mesh construction, so we focus on tissues having a spherical topology in the head. Then, let $I_{4}$ be the simplified image containing 4 tissues of the segmented child head (see Figure 2 (a)): the brain (composed of white and grey matter, blood vessels in the brain), CSF, bones and scalp (composed of skin, eyes, muscles, fat ...). Each head structure is numbered from 1 to 4 , from the most interior to the most exterior one (see in Figure 2 (b) the topological scheme of the 4 considered structures). The structure 0 corresponds to the background of the image.

To build our tetrahedral model, we use the method exposed in [3]. Indeed, this method uses good quality meshes called Almost Regular Tesselation and the labeling guarantees a spherical topology for the anatomical structures. In this paper (Section 3.3), we propose 


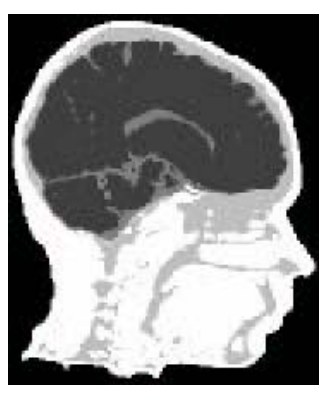

(a)

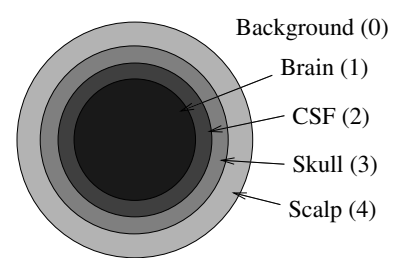

(b)
Figure 2: Simplified segmented image (one slice of the $3 \mathrm{D}$ volume) with 4 structures (a) and associated topological scheme (b).

some modifications of the original method in order to solve some geometrical problems.

\subsection{Almost Regular Tesselation}

Here we present a tetrahedral mesh called Almost Regular Tesselation (ART) (see $[10,11]$ for more details).

A tetrahedral tesselation of $\mathbb{R}^{3}$ is Almost Regular if it is possible to tessellate $\mathbb{R}^{3}$ with tetrahedra such that each tetrahedron has a fixed connectivity. The principle of our ART construction is based on the following notion: a tetrahedron $T$ is said to be subdivision invariant (SI) if we can divide $T$ into 8 tetrahedra which are congruent to $T$ (scaled by a factor $1 / 2$ ) by halving the edges of $T$. In an ART, a vertex is shared by 24 tetrahedra. Note that the regular tetrahedron is not subdivision invariant. Figure 3 (a) shows an example of SI tetrahedron.

As shown in [11], to obtain a tesselation as regular as possible, we choose the following SI tetrahedron which has an optimal quality:

$$
T^{*}=\left\{\left(\begin{array}{l}
0 \\
0 \\
0
\end{array}\right),\left(\begin{array}{l}
1 \\
0 \\
0
\end{array}\right),\left(\begin{array}{c}
\frac{1}{3} \\
\frac{2 \sqrt{2}}{3} \\
0
\end{array}\right),\left(\begin{array}{c}
\frac{2}{3} \\
\frac{\sqrt{2}}{3} \\
\frac{2}{3}
\end{array}\right)\right\}
$$

We build the ART initialization as the polyhedron $P$ composed of 24 tetrahedra $T^{*}$ sharing a common vertex. This vertex is chosen as the center of the segmented head, and we use a scale factor $S$ to enlarge $P$ until it contains entirely the head. Next, each tetrahedron is recursively subdivided $n$ times until the desired precision is reached (see Figure $3(\mathrm{~b})$ ). The final number of tetrahedra of the ART is $N_{n}=24 * 8^{n}$
$\left(N_{4}=98304\right.$ and $\left.N_{5}=786432\right)$. Moreover each tetrahedron of the ART has the same quality as $T^{*}$.

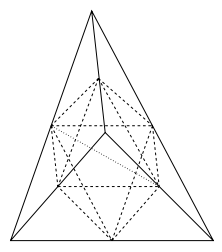

(a)
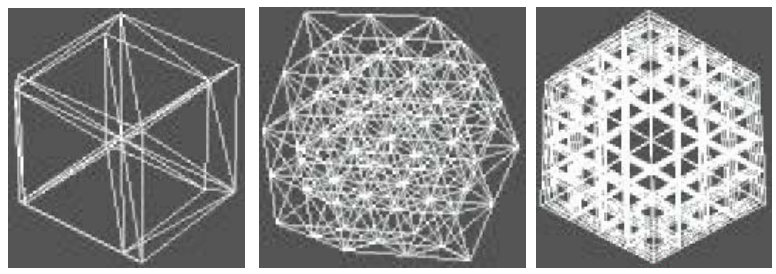

(b)

Figure 3: An SI tetrahedron and its subdivisions into 8 tetrahedra (a) and an ART construction (b) with from left to right: $n=0, n=1$ and $n=2$.

\subsection{Initial labeling method description}

The process described in [3] allows to label tetrahedra of an ART, according to their belonging to the considered structures segmented in the input image. It uses topological tools guaranteeing a spherical topology for the head. In this Section, we briefly describe this method and we show some obtained results from $I_{4}$.

First we associate to each tetrahedron $t$ of the ART a vector $T a b(t)$ of size 5 containing the percentages of each segmented component (background or tissue) in $t$. These vectors are computed thanks to $I_{4}$. The value $\operatorname{Tab}(t)[0]$ corresponds to the percentage of background in $t, \operatorname{Tab}(t)[1]$ to the percentage of brain and so on. For instance, if $\operatorname{Tab}(t)=[0.00,0.62,0.27,0.11,0.0]$, then $t$ contains $0 \%$ of background, $62 \%$ of brain, $27 \%$ of CSF, $11 \%$ of skull and $0 \%$ of scalp. We denote $P_{\text {Head }}(t)=$ $\sum_{i=1}^{n_{t}} \operatorname{Tab}(t)[i]=1-\operatorname{Tab}(t)[0]$.

The labeling uses the notion of simple tetrahedron. Such a tetrahedron can be removed from a set of tetrahedra $X$ without changing the topology of $X$. In [10], a local characterization of the simplicity of a tetrahedron 
is proposed, allowing us to define algorithms based on the use of simple tetrahedra. A set of tetrahedra $X_{1}$ is said to be homotopic to $X_{2}$ if we can obtain $X_{1}$ from $X_{2}$ by a sequential deletion (resp. addition) of simple tetrahedra.

A first step of the method is the generation of the set $H$ of tetrahedra belonging to the head, in opposition with the tetrahedra that belong to the background. To obtain $H$, we choose a tetrahedron $t$ such that $\operatorname{Tab}(t)=$ $[0.00,1.00,0.00,0.00,0.00]$ ( $t$ contains only brain, and so we are sure it belongs to $H$ ). Then we set $H=$ $\{t\}$ and we sequentially add to $H$ simple tetrahedra $x$ such that $P_{\text {Head }}(x)>\mu_{H}$, with $0 \leq \mu_{H}<1\left(\mu_{H}\right.$ is a parameter of the method). The final set $H$ is homotopic to a set made of a single tetrahedron, i.e. a full sphere.

Now that we have $H$, let us describe the method of [3] illustrated in Figure 4. The idea is to first label structures having an empty sphere topology as the scalp, skull or CSF (resp. tissues 4, 3 and 2), by topologically deforming a set having also an empty sphere topology. We label the tissues $i$ from the most exterior one to the most interior one. Then we start with $i=4$.

Let $O$ be a set of tetrahedra. Initially we have $O=H$. We choose a tetrahedron $t$ in the interior of $O$. Then we apply an interior thinning to the set $O \backslash t$ : starting from $t$, we remove in $O \backslash t$ until stability simple tetrahedra $x$ such that $\operatorname{Tab}(x)[4]<\mu_{i}$, where the parameter $\mu_{i}$ satisfies $0<\mu_{i} \leq 1$ (see Figure 4 (a)). The set $R$ of unremoved tetrahedra corresponds to the tissue $i$, and so all tetrahedra of $R$ are labeled with $i=4$.

Next $O$ becomes $O \backslash R, i$ becomes $i-1$, and we apply this process again while $i>1$ (see Figure $4(\mathrm{~b})$ ). The final set $O \backslash R$ corresponds to the tissue $i=1$ (the final $R$ corresponds to the tissue $i=2$ ).

By applying this labeling method to $I_{4}$, we obtain the results in Figure 5, with $n=5, S=170 \mathrm{~mm}$ and $\mu_{i}=0.5$ for $1 \leq i \leq 4$. We can remark that this method provides a tetrahedral mesh satisfying a spherical topology, even if the input segmented image does not (see Figure 6: sometimes the width of the CSF can be thinner than the voxelic resolution, especially in children MRI). But we need to modify the method in order to provide also geometrically correct meshes (see the jaw, for example, which is split, or missing isolated components for the bones).

\subsection{Labeled mesh corrections}

In the reality, the scalp bounds the bones, the skull bounds the CSF, and the CSF bounds the brain. But the topology of these structures is more complicated
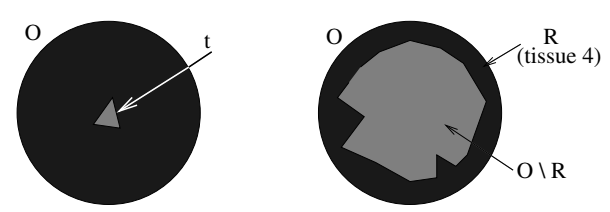

(a)
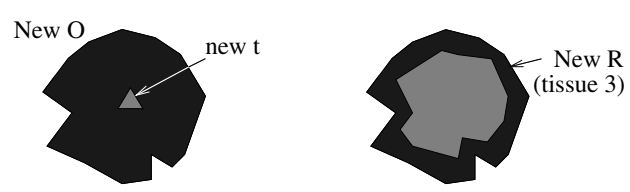

(b)

Figure 4: Labeling of tissues $i$, with $i>1$.

than empty or full spheres. To correct the geometry of the labeled mesh, the idea is to assure a minimal spher$i$ cal component for each structure $i>1$ when needed. Indeed, for each head component $i>1$, we want that one subset of the set labeled with $i$ has an empty sphere topology and bounds the component $i-1$. Then we authorize the change of label for tetrahedra that do not belong to this spherical component.

\section{The scalp and the bones}

To complete the skull, and the bones in general, we first label the scalp and the bones using the method proposed in Section 3.2. Let $S$ be the set of tetrahedra labeled with $i \in\{3,4\}$. Some tetrahedra labeled with $i=4$ (scalp) should be labeled with $i=3$ (bones). Then, as shown in Figure 7 , we define a minimal set of tetrahedra $M \subset S$ as the exterior border of the scalp where any change of label is forbidden. This zone guarantees that a hole will never appear through the scalp between the bones and the background. Next, we authorize the change of label from $i=4$ to $i=3$ if $T a b[3]>T a b[4]$. The result of this strategy is shown in Figure 8.

Next we consider the two other structures.

\section{The CSF and the brain}

Due to the cortex folding, the spatial arrangement of CSF and brain is very complex, and the CSF can be very thin. We propose two possibilities:

- first, we consider that as in the reality, the CSF bounds the brain and so we define a minimal layer 

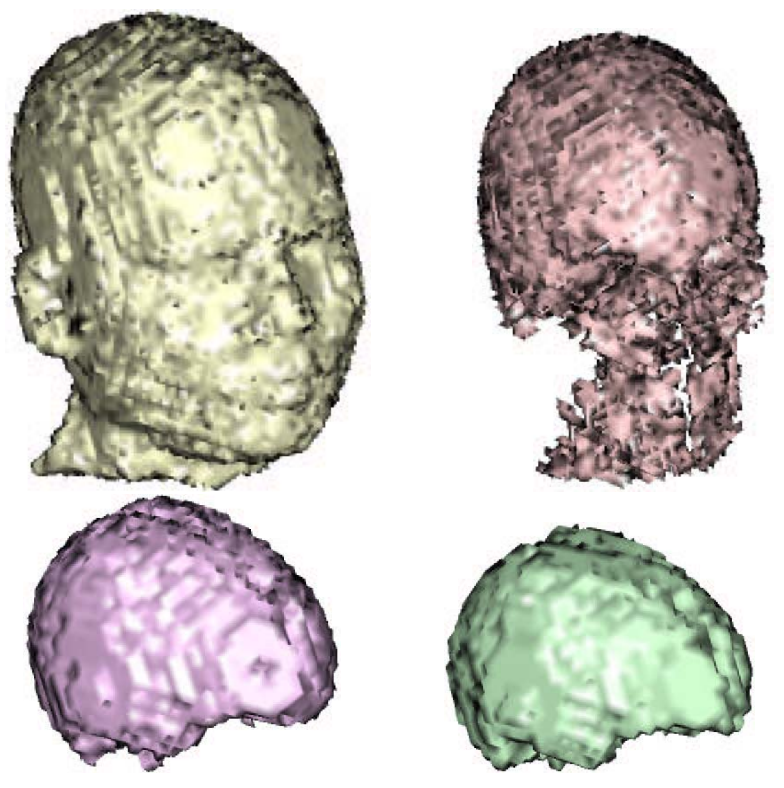

Figure 5: Result of the algorithm of [3] applied to $I_{4}$ (from top to bottom and left to right: exterior surfaces of the scalp, bones, CSF and brain).

of CSF in the tetrahedral mesh between the skull and the brain; this layer is obtained by applying an interior thinning (see Figure 4) in which we remove all simple tetrahedra. Then we label the other tetrahedra according to the majority structure in each tetrahedron for $i \in\{1,2\}$;

- a second possibility is to label the whole tetrahedra according to the majority structure in each tetrahedra for $i \in\{1,2\}$.

The results of the first strategy are shown in Figure 9. We can see that there is a minimal layer of CSF having a spherical topology (there are no more holes in the exterior layer of the CSF), and there are also isolated components of CSF in the interior of this layer.

The results of the second strategy are shown in Figure 10. The CSF is only composed of several components: places in the segmented image where CSF was too thin are missing.

\section{Conclusions and perspectives}

Despite the anatomical variations between different individuals (adults and children) and the diversity of the input images (acquired by several scanners in various hospitals), we have observed a satisfactory insensitivity
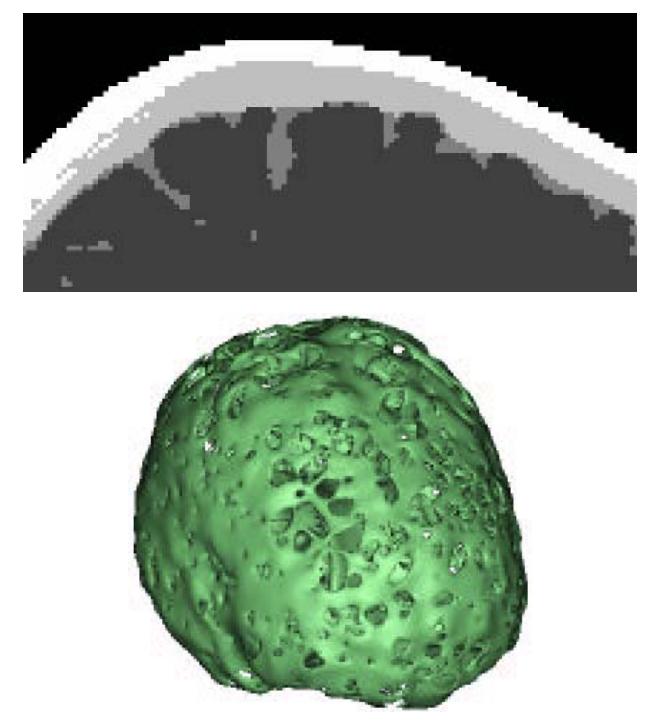

Figure 6: Zoom on the segmented image of Figure 2 (top) and extracted surface of the CSF (bottom): the CSF is very thin and, in the segmented volume, the brain (dark grey) touches the skull (light grey) through holes in the CSF. This situation is automatically corrected by the proposed mesh labeling procedure.

to the parameters, thus limiting the necessary manual intervention. In some instances one may need to adjust some thresholds to obtain reasonable estimates of the different structures volumes. However, the identification of the skull (for example) on a $256 \times 256 \times$ 130 volume (i.e. equal to $9 \mathrm{MB}$ of data if coded on 8 bits) takes less than one minute of processing time on a $1 \mathrm{GHz}$ Pentium III processor. The segmentation result is always a partition of the space, i.e. no points remain unlabeled or associated to two structures. This method is currently intensively tested on numerous child head images with different ages.

Concerning the realistic tetrahedral mesh construction from a simplified version of the MRI segmentation, our method partly uses the method exposed in [3]. By modifying this initial algorithm, we assure a minimal spherical topology for the scalp, the skull and eventually the CSF. Moreover, we provide results with geometrically shapes adapted to complex segmented head images. Our meshes are also perfectly adapted to the application of numerical methods computing the electromagnetic field propagation: good quality tetrahedra and correct topology for the anatomical structures. A perspective to the mesh generation is to take into account, from the segmented head, more than the four considered tissues in this paper. Indeed the aim is to 


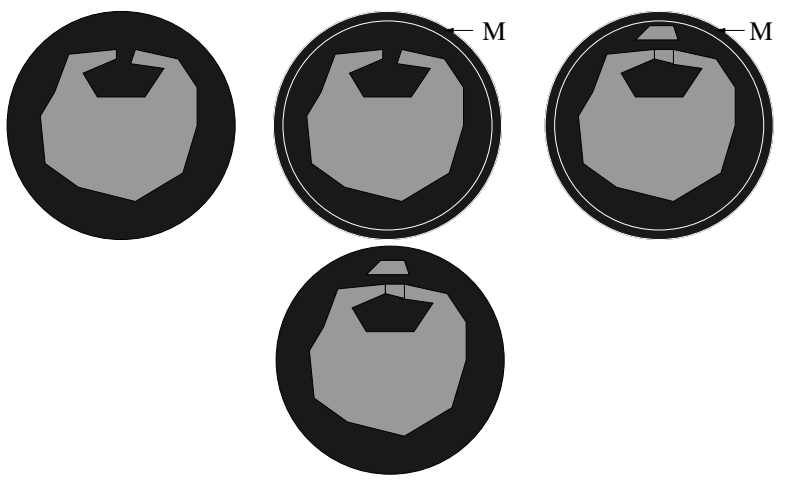

Figure 7: Scheme of the correction method for the bones (dark grey: scalp, light grey: bones).

build tetrahedral head models as realistic as possible.

\section{Acknowledgments}

This work has been partially supported by a grant from INRIA (ARC Headexp) and by a grant from the French ministry of research (RNRT Adonis).

\section{References}

[1] S. Beucher. Watershed, hierarchical segmentation and waterfall algorithm. In J. Serra and P. Soille, editors, Mathematical Morphology and Its Application to Image Processing, volume 2, 1994.

[2] E. J. Breen and R. Jones. Attribute opening thinnings and graulometries. Computer Vision and Image Understanding, pages 377-389, 1996.

[3] J. Burguet and I. Bloch. Homotopic labeling of elements in a tetrahedral mesh for the head modeling. Technical report, Ecole Nationale Supérieure des Télécommunications, apr 2004.

[4] P. Dokladal, I. Bloch, M. Couprie, D. Ruijters, R. Urtasun, and L. Garnero. Topologically Controlled Segmentation of 3D Magnetic Resonance Images of the Head by using Morphological Operators. Pattern Recognition, 36(10):2463-2478, oct 2003 .

[5] P.L. George and E. Séveno. The Advancing Front Mesh Method Revisited. Internation Journal in Numerical Methods in Engineering, 37:3605-3619, 1994.
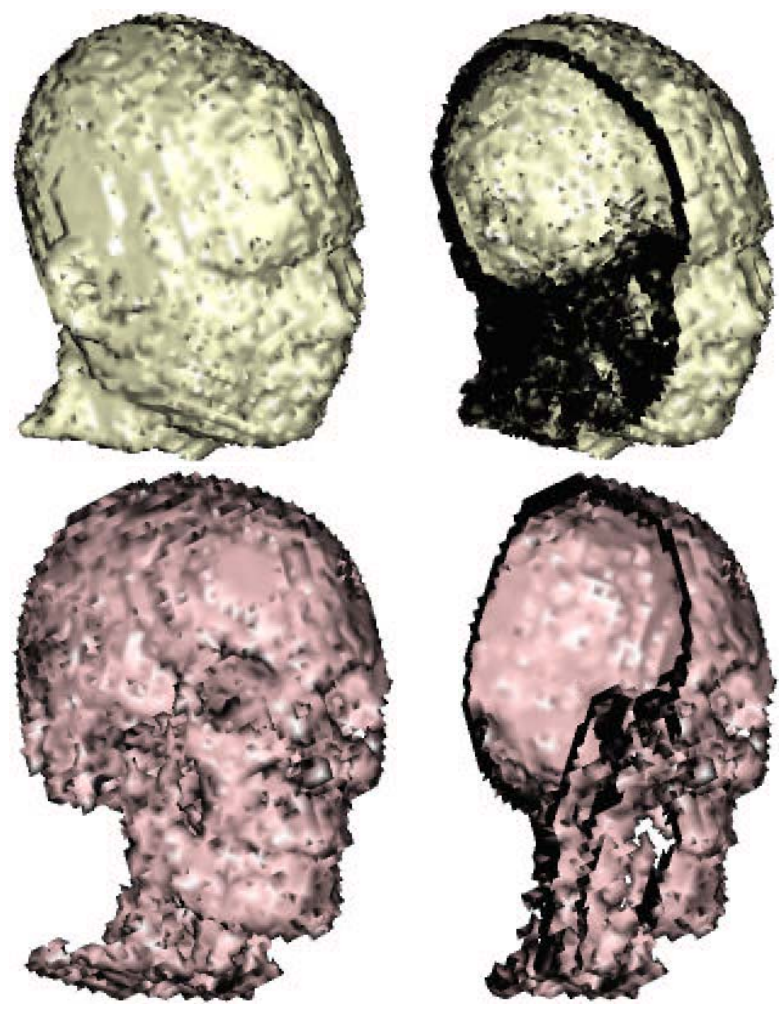

Figure 8: Result of the correction of the scalp (top) and the bones (bottom): the jaw is completed and isolated components may appear (right part: cut scalp and bones).

[6] P. Hanusse and P. Guillataud. Semantique des imagespar analyse dendronique. In 8th Conf Reconnaissance des Formes et Intelligence Artificielle, pages 577-588, Lyon, France, 1992.

[7] J. Mattes and J. Demongeot. Efficient algorithms to implement the confinement tree. In Comp Science, pages 392-405, 2000.

[8] J.C.D. Munck. The Potential Distribution in a Layered Anisotropic Spheroidal Volume Conductor. Journal of applied Physics, 64:464-470, 1988.

[9] J. Peraire and K. Morgan. Unstructured Mesh Generation Including Directional Refinement for Aerodynamics Flow Simulation. Finite Elements in Analysis and Design, 25(3-4):343-355, 1997.

[10] J. Pescatore. Maillages Homotopiques Tétraédriques des Tissus de la Tête pour le Calcul du Problème Direct en Électro/Magneto- 

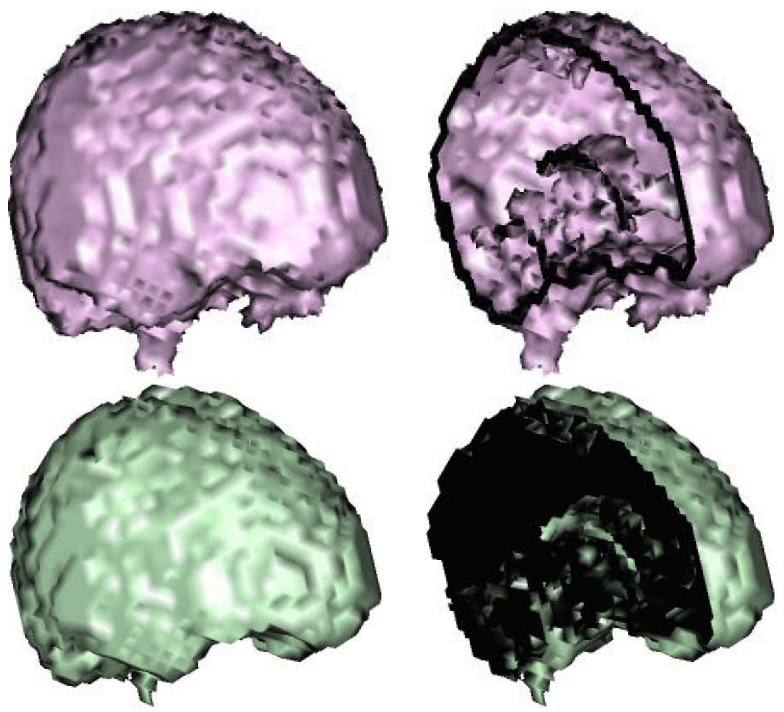

Figure 9: Result of the labeling of the CSF (top) and the brain (bottom) guaranteeing a minimal spherical component for the CSF.

Encéphalographie. $\mathrm{PhD}$ thesis, École Nationale Supérieure des Télécommunications, 2001.

[11] J. Pescatore, I. Bloch, S. Baillet, and L. Garnero. FEM Tetrahedral Mesh of Head Tissues from MRI under Geometric and Topological Constraints for Applications in EEG and MEG. In Human Brain Mapping HBM 2001, page 218, Brighton, UK, jun 2001.

[12] J. Sarvas. Basic Mathematical and Electromagnetic Concepts of the Biomagnetics Inverse Problem. Physics in Medical Biology, 32:11-22, 1987.

[13] L. Vincent and P. Soille. Watershed in digital spaces, an efficient algorithm based on immersion simulation. IEEE Transactions on Pattern Analysis and Machine Intelligence, 13(6), jun 1991.
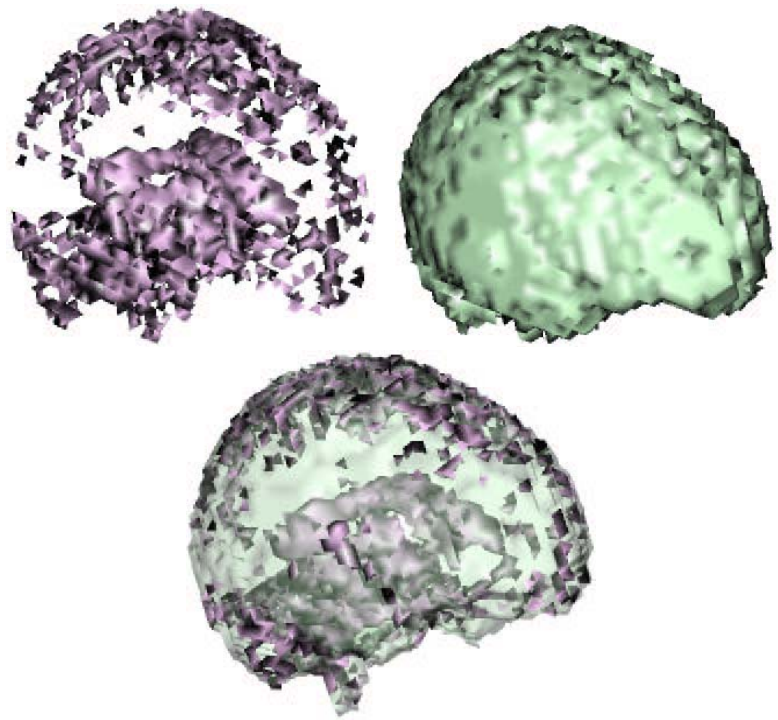

Figure 10: Result of the labeling of the CSF (top left) and the brain (top right) and the CSF together with the brain. The tetrahedra are labeled according to the majority structure. 